\title{
GENERAL FEATURES AND OUTCOMES OF ADULT PATIENTS WITH DERMATOMYOSITIS: A PROSPECTIVE ONE-CENTER COHORT STUDY
}

\author{
Natássia Cristina Carboni Truzzi ${ }^{1, \star}$, Leonardo Santos Hoff ${ }^{1}$, Isabela Bruna Pires Borges ${ }^{1}$, Maria Aurora Gomes da Silva ${ }^{1}$, Samuel \\ Katsuyuki Shinjo ${ }^{1}$
}

1.Universidade de São Paulo, São Paulo (SP), Brazil.

*Corresponding author: n.atassia@hotmail.com

\section{BACKGROUND}

Currently, studies that have prospectively analyzed a large sample of patients with dermatomyositis (DM) are scarce. The majority of available studies about DM are based on case reports, case series, and retrospective cohorts, or they included different types of myositis or probable/possible DM. Therefore, we aimed to assess a large sample of patients with defined DM prospectively.

\section{METHODS}

This prospective, one-center cohort study included 91 adults with defined classical DM (EULAR/ACR 2017) who have been followed up in our outgoing clinic since 2012. Patients with overlapping syndromes or clinically amyopathic DM were excluded. Autoantibodies were assessed by immunoblotting antibody assay.

\section{RESULTS}

The mean age of patients was $47.3 \pm 15.4,67.0 \%$ were women, and $81.3 \%$ were white. The median follow-up time was 44 (IQR 17-67) months, and $82.4 \%$ of patients attended their follow-up appointments. As initial treatment, $22.2 \%$ of patients received methylprednisolone pulse therapy ( $3-5 \mathrm{~g}$ ), received $4.4 \%$ intravenous immunoglobulin, and $54.9 \%$ received both drugs. Currently, $67.0 \%$ of patients are under immunosuppressive drugs or rituximab, and $33.0 \%$ are still taking glucocorticoid (median dose of 15 [7.5-40] mg/day). One-third of patients had disease remission, $48.4 \%$ had complete clinical response, and $18.7 \%$ saw continued disease activity. Concerning comorbidities, $46.7 \%$ of patients had arterial hypertension, $20.0 \%$ had diabetes mellitus, and $4.4 \%$ had myocardial infarction. During follow-up, 102 infections occurred in 48 patients (15 pneumonia, 11 zoster, 5 tuberculosis, 3 COVID-19, and 68 others). Malign neoplasia was identified in 22 cases ( 8 cases before, 10 during, and 3 after DM diagnosis). The most common was breast cancer $(n=9)$ followed by 4 gastric, and 2 lung cancers. In total, $9.9 \%$ of patients died (sepsis, pneumonia, and neoplasia). We observed 13.5\% anti-Mi-2, 13.5\% anti-Jo-1, 12.2\% anti-MDA-5, 9.5\% anti-TIF1, 6.8\% anti-NXP-2, 8.1\% anti-SAE, 2.7\% anti-PL7, 1.4\% anti-PL-12,1.4\% anti-SRP, and 35.1\% anti-Ro-52, and 5.5\% anti-PM/SCl, and no cases of anti-Ku, anti-EJ, and anti-OJ. ANA was present in $83.8 \%$ of the cases. Only moderate correlation was seen among anti-Jo- 1 and lung involvement. Fair correlations were observed among anti-TIF-1 and the risk of pneumonia and neoplasia, anti-Mi-2 and increase of muscle enzymes, and anti-MDA-5 with cutaneous ulcers and vasculitis.

\section{CONCLUSION}

This study presents the general profile of Brazilian patients with DM. Most patients received both methylprednisolone pulse therapy and intravenous immunoglobulin, which may have contributed to the favorable outcome for almost half of them. Notwithstanding, the prevalence of infections and cardiovascular diseases was high.

\section{FUNDING}

FAPESP Grant No. 2021/04892-0 to NCCT, and 2019/11776-6 to SKS.

\section{KEYWORDS}

Dermatomyositis, Autoantibodies, Treatment. 